\title{
Application of equivalent cylindrical inhomogeneity to modeling of CNT and analysis of influence of CNT distributions on response of functionally graded structural elements
}

\author{
Lidiia Nazarenko ${ }^{1}$, Aleksandr Chirkov ${ }^{2}$, Henryk Stolarski ${ }^{3}$, Holm Altenbach ${ }^{4}$ \\ ${ }^{1,4}$ Institute of Mechanics, Otto von Guericke University Magdeburg, Germany \\ ${ }^{2}$ Pisarenko Institute of Problems of Strength, NAS of Ukraine, Kyiv, Ukraine \\ ${ }^{3}$ Department of Civil, Environmental and Geo- Engineering, University of Minnesota, Minneapolis, USA
}

E-mail: 'lidiia.nazarenko@ovgu.de (corresponding author)

\begin{abstract}
A mathematical model based on the concept of the energy-equivalent inhomogeneity combined with the method of conditional moments (MCM) has been applied to analyze Carbon Nanotube (CNT)-reinforced materials. The idea of the energy-equivalent inhomogeneity is to replace the inhomogeneity and its interphase by a single equivalent inhomogeneity. It is evaluated for cylindrical inhomogeneity with Gurtin-Murdoch surface model and spring layer model of interphases. Inclusion of multiple mechanisms in the description of interphases is applied to the CNT-reinforced materials. The CNT is modeled as a cylindrical high-stiffness surface. A weak zone surrounding the CNT can be modeled by a spring layer. In this case, one Gurtin-Murdoch and one spring layer models would need to be combined. To evaluate the effective properties of CNT-reinforced materials a statistical method, the MCM, has been employed which describes random distribution of CNT's. Closed-form formulas for the components of the effective stiffness tensor of such composites have been developed. A composite plate weakened by a hole under different types of loading is considered. It is assumed, that the plate made of the polymer with randomly distributed and disoriented CNT's. Analysis of how various special distributions of CNT concentration affect the response of this plate is performed.
\end{abstract}

Keywords: carbon nanotubes, Gurtin-Murdoch surface model, spring layer model, functionally graded materials.

\section{Introduction}

Importance and relevance of carbon nanotubes (CNT's) as a reinforcement are well known due to their attractive mechanical, thermal and electrical properties. Evaluation of the effective properties of random carbon nanotubes reinforced materials (CNTRM's) is currently handled using usual techniques of micromechanics such as composite sphere assemblage of Hashin $(1990,1991)$ or Mori-Tanaka method (e.g., Seidel \& Lagoudas, 2006), or computer-based methods (e.g., Georgantzinos et al., or Tserpes and Papanikos, in Tserpes and Sylvester (Eds.) 2014). The second assumes, as a rule, a regular structure of the composite and involves numerical analysis of a unit cell containing a single CNT. The solution of such problem is processed to obtain the effective properties for material with any prescribed distribution of CNT orientations (e.g. Song \& Youn, 2006). Those two approaches, while providing useful results, are not optimal for determination of the effective properties of CNT-reinforced, random materials.

The approximate approach for analysis of CNTRM's was recently developed in Nazarenko, Stolarski, and Altenbach (2018b) and it is based on probabilistic approach. In this approach, the Gurtin-Murdoch (GM) theory (Gurtin \& Murdoch, 1975) is applied to a hollow cylinder of finite thickness (Nazarenko et al. 2016, 2018b) and combined with the notion of energy equivalence (Nazarenko \& Stolarski, 2016a) to replace CNT by an equivalent solid cylinder. The interphase is modeled as a spring layer and is subsequently added to obtain another equivalent solid cylinder. Effective properties of CNRTM are evaluated using the method of conditional moments (MCM) originally developed in Nazarenko, Khoroshun, Müller, and Wille (2009), Nazarenko, Stolarski, and Altenbach (2018a). In addition, it leads to a sequence of the closed-form expressions. It is very attractive feature, which can be useful in design of structural elements made of CNTRM's with monotonously changing mechanical properties.

In recent years, considerable attention has been focused on the functionally graded material (FGM) - artificially produced particulate composites with smoothly spatially varying mechanical, thermal and electrical properties, and a number of reviews dealing with various issues of FGM's have been published (e.g., Birman \& Byrd, 2009; Xu, X. Zhang, \& M. Zhang, 2018). The most of research studies in FGM's had focused on stress analysis, fracture mechanics and optimization problems (Birman \& Byrd, 2009; Grigorenko, Dyyak, \& Makar, 2001; L. Li, X. Li, \& Hu, 2016

(C) 2019 Authors. Published by VGTU Press. This is an open-access article distributed under the terms of the Creative Commons Attribution (http://creativecommons.org/licenses/by/4.0/) License, which permits unrestricted use, distribution, and reproduction in any medium, provided the original author and source are credited. 
among others). Potential advantages of such materials are the elimination of stress discontinuity, producing the optimal set of properties and mitigating stress concentrations in the dangerous zones of structural elements.

The goal of the present work is to apply the above methodology to modelling and analysis of structural elements made from CNTRM's under assumption that CNT volume fraction varies smoothly or piecewise in the zone of stress concentration. CNTRM considered here is treated, in a sense, like FGM.

\section{Modeling of carbon nanotubes reinforced materials (CNT)}

The general methodology presented in this work for modelling of CNT consists of replacing the original inhomogeneity and the surrounding domain by uniform equivalent inhomogeneity, which is perfectly bonded to the matrix. The properties of the equivalent inhomogeneity are obtained from the assumption that the energies of the inhomogeneity/interphase system and of the uniform equivalent inhomogeneity are equal for arbitrary displacements on the system boundary corresponding to arbitrary constant strain within its volume. That way the composites including interphases can be analyzed using techniques available for composites without interphases.

In accordance with above concept, we consider CNT as a thin hollow cylinder of finite length modeled as a GM (Gurtin \& Murdoch, 1975) material surface. In this case, the surface properties of the CNT should be used as the interphase properties (Nazarenko et al., 2016, 2018b).

$\mathrm{CNT} /$ matrix interphase can be included using the concept of energy equivalence hierarchically one more time (Nazarenko \& Stolarski, 2016b) where the interphase can be described using the spring layer model (Nazarenko \& Stolarski, 2016a; Nazarenko, Stolarski, \& Altenbach, 2017). This would lead to the final solid equivalent cylinder combining properties of the CNT and of its interphase. The formulas developed in the papers Nazarenko et al. (2016, 2017) for the stiffness tensor of the equivalent solid cylindrical fiber with GM material surface model and spring layer model of interphase are quite involved, and the Reader is referred to the original paper for details.

Using energy equivalent inhomogeneity approach for modelling of CNT with interphase the effective properties of CNT reinforced materials accounting for interphases can be evaluated by the method of conditional moments (MCM) as described in Nazarenko et al. $(2016,2017)$ in the context of the materials reinforced with randomly distributed unidirectional short solid fibers. In the case of CNT reinforced materials the short solid cylinders equivalently represent CNT. Such composite has transversely isotropic symmetry of elastic properties in the macro volume. Closedform relations for five independent components of the tensor of effective moduli are presented in Nazarenko et al. (2009).

\section{Analysis of strain concentration of functionally graded plate with hole made from CNT reinforced polymer}

In order to demonstrate how the outlined above approach can be applied to analysis of influence of CNT distribution on overall response of structural elements we consider representative numerical example of square plate with hole made from CNT reinforced polymer.

In Figure 1 it is schematically illustrated the square plate where length of the edges is $l=10 \mathrm{~cm}$, thickness $h=1 \mathrm{~cm}$, and hole of diameter $-d_{a}=1 \mathrm{~cm}$.

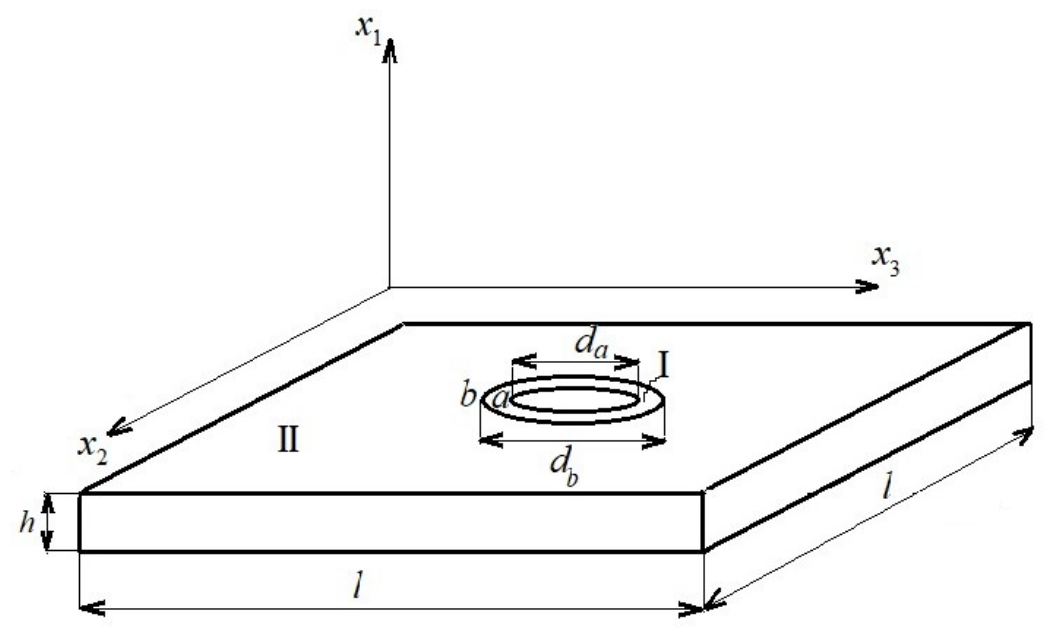

Figure 1. Schematic illustration of the plate with hole

The material of the plate is polymer isotropic matrix $\left(E_{\mathrm{m}}=2.026 \mathrm{GPa}, v_{\mathrm{m}}=0.3\right)$ with randomly distributed and completely disoriented CNT's. CNT is modelled as a thick hollow cylinder of finite length. We assume that the 
CNT is isotropic with the three-dimensional Young's modulus $E_{\mathrm{CNT}}=1100 \mathrm{GPa}$ and the Poisson's ratio $v_{\mathrm{CNT}}=0.14$. The outer radius of the CNT is $r_{\mathrm{o}}=0.85 \mathrm{~nm}$ and their thickness is $h=0.34 \mathrm{~nm}$. The properties of solid cylinder, which is equivalent to the CNT are determined in Nazarenko et al. (2018b), here we present just the final results for stifness tensor of this cylinder

$$
\begin{gathered}
E_{\mathrm{eq}[1]}=244.24 \mathrm{GPa}, E_{\mathrm{eq}[3]}=718.11 \mathrm{GPa} \\
v_{\mathrm{eq}[13]}=0.1625, v_{\mathrm{eq}[12]}=0.3241, \mu_{\mathrm{eq}[12]}=92,44 \mathrm{GPa} .
\end{gathered}
$$

In addition, we assume that distribution of the CNT volume fraction in the ring around the plate hole with outer diameter $d_{b}=2 \mathrm{~cm}$ (zone I in Figure 1) is different than in the rest part of the plate (II in Figure 1). We consider five cases of different distributions of CNT volume fraction in two parts of the plate but in all cases CNT volume fraction averaged over the entire plate is the same and equal to $\bar{c}_{\mathrm{CNT}}=0.1$.

Case 1. Value of the CNT volume fraction increases monotonously along radius in the ring around hole (zone I) from 0 at the inner circle of the hole $c_{\mathrm{CNTI} a}=0.0$ up to $c_{\mathrm{CNTI}}=0.101$ at the outer circle of the ring around hole. The rest part of the plate (zone II) has constant CNT volume fraction equal to $c_{\mathrm{CNTII}}=0.101$.

The effective properties of CNT reinforced polymer with randomly distributed and completely disoriented CNT's are determined by the MCM (Nazarenko et al., 2009) and then disoriented in accordance to approach presented in Khoroshun and Nazarenko (1992).

The effective Lamé parameters of material I in point $a$ (inner circle of the ring around hole) and in point $b$ (outer circle of the ring around hole) are

$$
\lambda_{I a}^{*}=1.1687 \mathrm{GPa}, \quad \mu_{I a}^{*}=0.77911 \mathrm{GPa}, \quad \lambda_{I b}^{*}=6.05829 \mathrm{GPa}, \quad \mu_{I b}^{*}=5.7127 \mathrm{GPa} .
$$

The effective Lamé parameters of material II (zone II) are

$$
\lambda_{I I}^{*}=6.05829 \mathrm{GPa}, \mu_{I I}^{*}=5.7127 \mathrm{GPa} .
$$

Case 2. Value of the CNT volume fraction decreases monotonously along radius in the ring around hole (zone I) from $c_{\mathrm{CNTI} a}=0.15$ at the inner circle of the hole up to $c_{\mathrm{CNT} I b}=0.0995$ at the outer circle of the ring around hole. The rest part of the plate (part II) has constant CNT volume fraction equal to $c_{\mathrm{CNTII}}=0.0995$.

The effective Lamé parameters of material I in point $a$ (inner circle of the ring around hole) and in point $b$ (outer circle of the ring around hole)

$$
\lambda_{I a}^{*}=8.4437 \mathrm{GPa}, \quad \mu_{I a}^{*}=8.1274 \mathrm{GPa}, \quad \lambda_{I b}^{*}=5.9869 \mathrm{GPa}, \quad \mu_{I b}^{*}=5.6405 \mathrm{GPa},
$$

and the effective Lamé parameters of material II (zone II)

$$
\lambda_{I I}^{*}=5.9869 \mathrm{GPa}, \mu_{I I}^{*}=5.6405 \mathrm{GPa} .
$$

Case 3. Value of the CNT volume fraction is constant in the ring around hole (zone I) and is equal to $c_{\mathrm{CNTIa}}=c_{\mathrm{CNTIb}}=0.0$. In the rest part of the plate (zone II) $\mathrm{CNT}$ volume fraction equal to $c_{\mathrm{CNTII}}=0.1024$.

The effective Lamé parameters of material I in point $a$ (inner circle of the ring around hole) and in point $b$ (outer circle of the ring around hole) are the same and are equal to

$$
\lambda_{I a}^{*}=\lambda_{I b}^{*}=1.1687 \mathrm{GPa}, \mu_{I a}^{*}=\mu_{I b}^{*}=0.77911 \mathrm{GPa} .
$$

The effective Lamé parameters of material II (zone II)

$$
\lambda_{I I}^{*}=6.1263 \mathrm{GPa}, \quad \mu_{I I}^{*}=5.7815 \mathrm{GPa} .
$$

Case 4. Value of the CNT volume fraction is constant in the ring around hole (part I) and equal to $c_{\mathrm{CNTIa}}=c_{\mathrm{CNTIb}}=0.15$. In the rest part of the plate (zone II) $\mathrm{CNT}$ volume fraction is equal to $c_{\mathrm{CNTII}}=0.09297$.

$$
c_{\mathrm{CNTI} a}=c_{\mathrm{CNTI} I b}=0.15, c_{\mathrm{CNTII}}=0.09297 .
$$

The effective Lamé parameters of material I in point $a$ (inner circle of the ring around hole) and in point $b$ (outer circle of the ring around hole) are the same and equal to

$$
\lambda_{I a}^{*}=\lambda_{I b}^{*}=8.4437 \mathrm{GPa}, \mu_{I a}^{*}=\mu_{I b}^{*}=8.1274 \mathrm{GPa} .
$$


Nazarenko, L.; Chirkov, A.; Stolarski, H.; Altenbach, H. 2019. Application of equivalent cylindrical inhomogeneity to modeling of CNT and analysis of influence of CNT distributions on response of functionally graded structural elements

The effective Lamé parameters of material II (zone II)

$$
\lambda_{I I}^{*}=5.6683 \mathrm{GPa}, \mu_{I I}^{*}=5.3184 \mathrm{GPa} .
$$

Case 5. As a reference case, we consider the plate with hole with constant CNT volume fraction and distribution in the entire plate, which are equal to 0.1

$$
\bar{c}_{\mathrm{CNT}}=c_{\mathrm{CNT} I a}=c_{\mathrm{CNT} I b}=c_{\mathrm{CNTII}}=0.1 .
$$

In this case effective Lamé parameters of material I and material II are the same and equal to

$$
\lambda_{I a}^{*}=\lambda_{I b}^{*}=\lambda_{I I}^{*}=6.0097 \mathrm{GPa}, \mu_{I a}^{*}=\mu_{I b}^{*}=\mu_{I I}^{*}=5.6635 \mathrm{GPa} .
$$

The effective Lamé parameters of CNT reinforced polymer for various volume fractions of CNT's in the interval from 0.0 to 0.15 are presented in Table 1 .

Table 1. Effective Lamé parameters of CNT reinforced polymer for various volume fractions of CNT's

\begin{tabular}{|c|c|c|c|c|c|}
\hline${ }^{c_{\mathrm{CNT}}}$ & $\lambda^{*}$ & $\mu^{*}$ & $c_{\mathrm{CNT}}$ & $\lambda^{*}$ & $\mu^{*}$ \\
\hline 0.000 & 1.168735 & 0.779138 & 0.080 & 5.038773 & 4.682401 \\
\hline 0.005 & 1.410006 & 1.022173 & 0.085 & 5.281370 & 4.927476 \\
\hline 0.010 & 1.651354 & 1.265323 & 0.090 & 5.524057 & 5.172695 \\
\hline 0.015 & 1.892782 & 1.508590 & 0.095 & 5.766834 & 5.418060 \\
\hline 0.020 & 2.134288 & 1.751974 & 0.100 & 6.009703 & 5.663574 \\
\hline 0.025 & 2.375874 & 1.995479 & 0.105 & 6.252664 & 5.909238 \\
\hline 0.030 & 2.617540 & 2.239104 & 0.110 & 6.495719 & 6.155055 \\
\hline 0.035 & 2.859288 & 2.482853 & 0.115 & 6.738868 & 6.401028 \\
\hline 0.040 & 3.101116 & 2.726725 & 0.120 & 6.982112 & 6.647159 \\
\hline 0.045 & 3.343028 & 2.970724 & 0.125 & 7.225454 & 6.893452 \\
\hline 0.050 & 3.585022 & 3.214851 & 0.130 & 7.468893 & 7.139908 \\
\hline 0.055 & 3.827100 & 3.459108 & 0.135 & 7.712431 & 7.386531 \\
\hline 0.060 & 4.069262 & 3.703496 & 0.140 & 7.956070 & 7.633324 \\
\hline 0.065 & 4.311510 & 3.948017 & 0.145 & 8.199810 & 7.880289 \\
\hline 0.070 & 4.553844 & 4.192674 & 0.150 & 8.443653 & 8.127430 \\
\hline 0.075 & 4.796265 & 4.437468 & & & \\
\hline
\end{tabular}

We consider the plate under biaxial tension in the $x_{2}$ and $x_{3}$ directions (Figure 1). The problem was solved in a two-dimensional formulation under the condition of a plane stress state. The solution of the problem is obtained by a mixed finite element method (FEM) using a mixed approximation on a triangular element (Figure 2). For details of the Reader is referred to the original publications (Chirkov, 2003a, 2003b).

$$
\sigma_{3}=100 \mathrm{MPa} ; \quad \sigma_{2}=100 \mathrm{MPa} .
$$

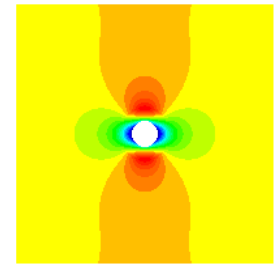

case 1

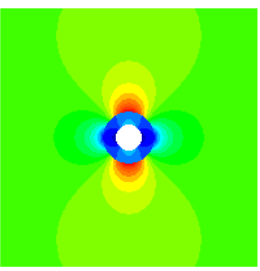

case 3
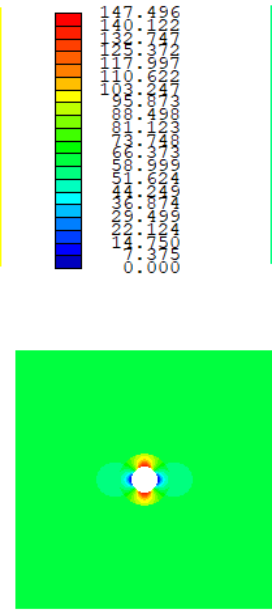

case 4

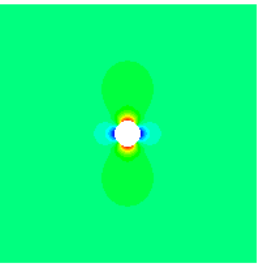

case 2

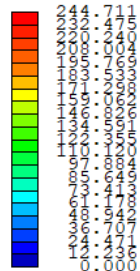

Figure 2. Stresses $\sigma_{3}$
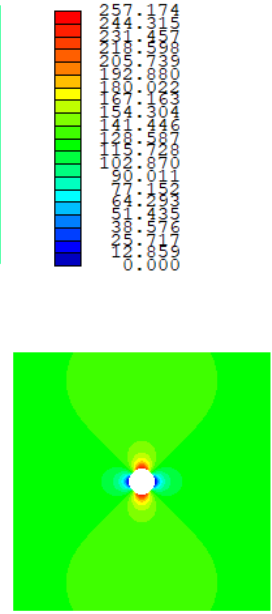

case 5

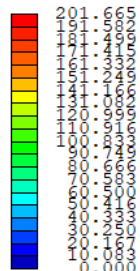

18: 0800 
The results of calculations for determining of axial stresses in loading direction $\sigma_{3}$ along axis $x_{2}$ are shown in Figures 2, 3 for the considered cases of distribution of CNT volume fraction. Maximal axial stresses $\sigma_{3}$ are on the boundary of hole along axis $x_{2}$ and they vary substantially for different cases. The minimal stresses are for case 1 where CNT volume fraction increases monotonously along radius from the inner to outer boundary of the ring around the hole (case 1). Maximum is obtained in the case if CNT volume fraction decreases monotonously along radius from the inner to outer boundary of the ring (case 2). The difference between these both cases is about $68 \%$. Intermediate values of the stress concentration correspond to the cases of piecewise and constant distributions of CNT volume fraction in the plate - 187.4 MPa (case 3), 201.7 MPa (case 5), 244.7 MPa (case 4). Moreover, in the case 1, stresses in loading direction $\sigma_{3}$ along axis $x_{2}$ are changed smoothly without jumps, what takes place in other cases of inhomogeneous distributions of CNT volume fraction.

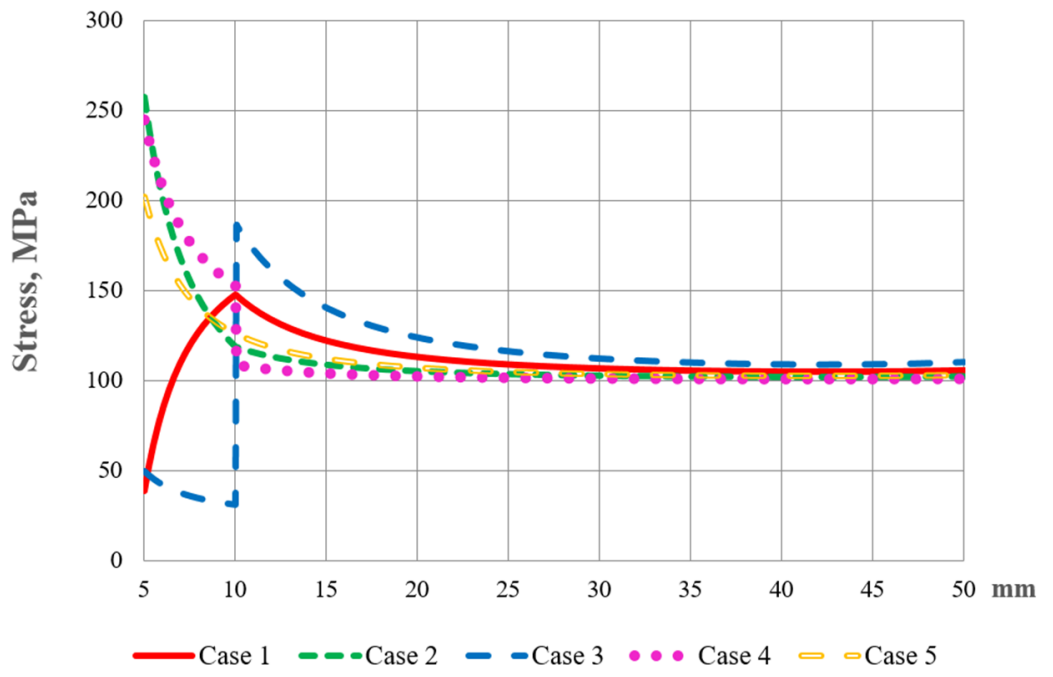

Figure 3. Dependence of stresses $\sigma_{3}$ along axis $x_{2}$ if $x_{3}=0$

The second numerical example used here for illustration of the influence of distribution of CNT volume fraction on the response of the plate with hole is the plate under tension in the in the $x_{3}$ direction and under compression in the perpendicular $x_{2}$ direction.

$$
\sigma_{3}=100 \mathrm{MPa} ; \quad \sigma_{2}=-100 \mathrm{MPa}
$$

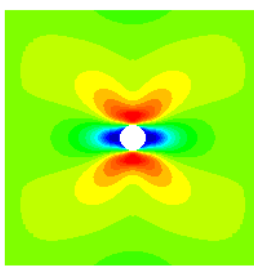

case 1
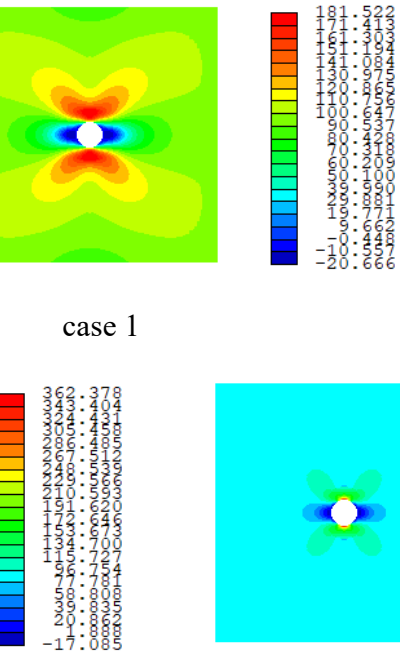

case 3

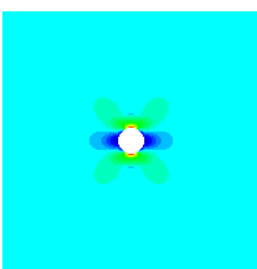

case 4

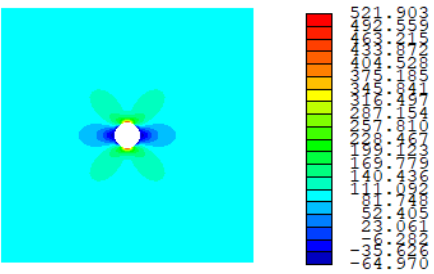

case 2

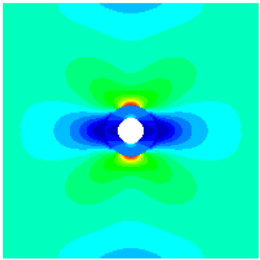

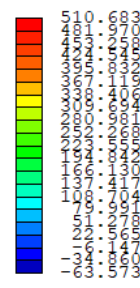

Figure 4. Stresses $\sigma_{3}$ 
The results for axial stresses in loading direction $\sigma_{3}$ along axis $x_{2}$ are presented in Figures 4, 5 for all cases of distribution of CNT volume fraction. Maximal axial stresses $\sigma_{3}$ are on the boundary of hole along axis $x_{2}$ and they vary even more substantially for different cases. The minimal stresses are for case 1 where CNT volume fraction increases monotonously along radius from the inner to outer boundary of the ring around the hole - about $181.5 \mathrm{MPa}$. Maximum is obtained in the case if CNT volume fraction decreases monotonously along radius from the inner to outer boundary of the ring (case 2) - 521.9 MPa. The difference between these both cases for such type of loaging is about $187.5 \%$. Intermediate values of the stress concen ration correspond to the cases of piecewise and constant distributions of CNT volume fraction in the plate - 362.4 MPa (case 3), 414.2 $\mathrm{MPa}$ (case 5), 510.5 MPa (case 4). In the case 1, as well as for the biaxial tension, stresses in loading direction $\sigma_{3}$ along axis $x_{2}$ are changed smoothly without jumps, what takes place in other cases of inhomogeneous distributions of CNT volume fraction.

The analysis of the plate with hole under different types of loading is a good illustration of that, how the proposed methodology can be applied to design of structural elements in order to reduce stress concentration in dangerous zone of construction.

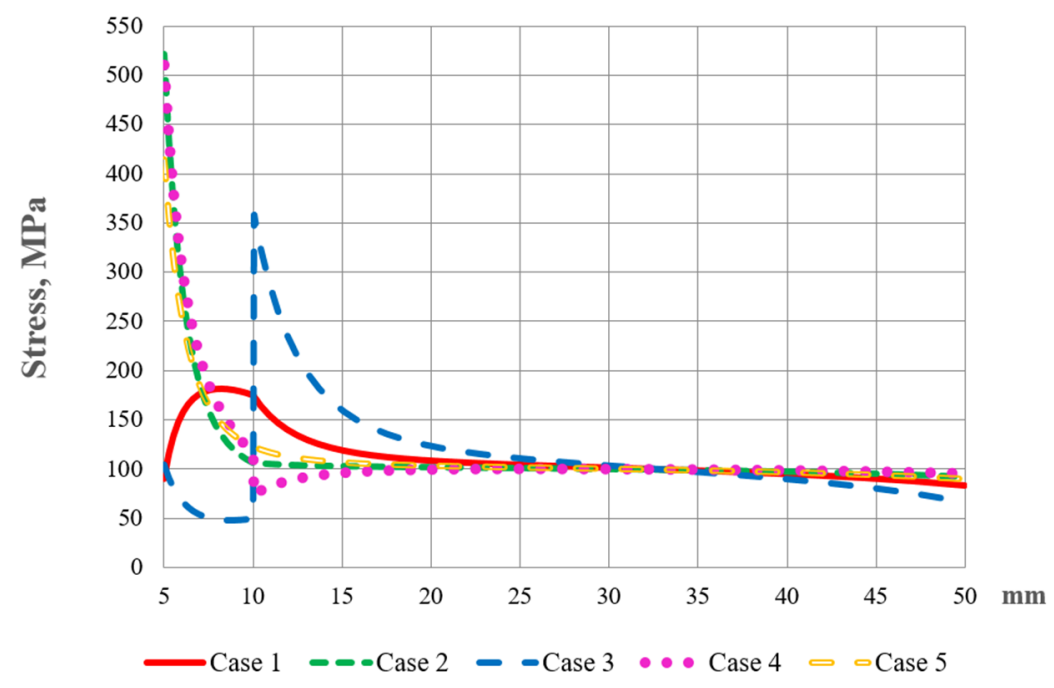

Figure 5. Dependence of stresses $\sigma_{3}$ along axis $x_{2}$ if $x_{3}=0$

\section{Conclusions}

It has been shown that the modeling of carbon nanotubes (CNT's) based on the notion of energy-equivalent inhomogeneity the Gurtin-Murdoch (membrane) model, in conjunction with method of conditional moments, is quite viable in evaluation of the effective properties of CNT reinforced materials and leads to closed-form relations. It is an attractive feature and can be successfully applied to analysis of structural elements made from such materials (functionally graded plate with hole made from CNT reinforced polymer under bi-axial loading) and to their optimization. This was the main goal of the present work.

Analysis of stress-strain of the plate with hole under bi-axial loading was provided in a two-dimensional formulation under assumption of the plane stress state. Mixed approximation of a triangular element was used in FEM calculations, what leads to determination of stresses with a high degree of accuracy.

It has been demonstrated that the variation of CNT volume fraction in the zone where maximal stress concentration appears under loading can reduce essentially the maximal stress concentration and by such way can advance the robustness of such structure.

\section{Acknowledgements}

LN and HS gratefully acknowledge the financial support by the German Research Foundation (DFG) via Projects NA1203/1-1 and NA1203/1-2. 
Nazarenko, L.; Chirkov, A.; Stolarski, H.; Altenbach, H. 2019. Application of equivalent cylindrical inhomogeneity to modeling of CNT and analysis of influence of CNT distributions on response of functionally graded structural elements

\section{References}

Birman, V., \& Byrd, L. W. (2009). Modeling and analysis functionally graded materials and structures. Applied Mechenical Review, 60(5), 195-216. https://doi.org/10.1115/1.2777164

Chirkov, A. Yu. (2003a). Mixed projection-mesh scheme of the finite-element method for the solution of problems of the elasticity theory. Strength of Materials, 35(3), 267-289. https://doi.org/10.1023/A:1024664806074

Chirkov, A. Yu. (2003b). Mixed approximation scheme of the finite-element method for the solution of two-dimensional problems of the elasticity theory. Strength Materials, 35(6), 608-632. https://doi.org/10.1023/B:STOM.0000013613.68456.30

Grigorenko, A. Ya., Dyyak, I. I., \& Makar, V. M. (2001). Influence of anisotropy on the response characteristics of finite cylinders under free vibrations. Internatinal Applied Mechanics, 37(5), 628-637. https://doi.org/10.1023/A:1012308412972

Gurtin, M. E., \& Murdoch, A. I. (1975). A continuum theory of elastic material surfaces. Archive for Rational Mechanics and Analysis, 57(4), 291-323. https://doi.org/10.1007/BF00261375

Hashin, Z. (1990). Thermoelastic properties of fiber composites with imperfect interface. Mechanics of Materials, 8(4), 333-348. https://doi.org/10.1016/0167-6636(90)90051-G

Hashin, Z. (1991). The spherical inclusion with imperfect interface. Journal of Applied Mechanics, 58(2), 444-449. https://doi.org/10.1115/1.2897205

Khoroshun, L. P., \& Nazarenko, L. V. (1992). Effective elastic properties of composites with disoriented anisotropic ellipsoidal inclusions. Internatinal Applied Mechanics, 28(12), 801-808. https://doi.org/10.1007/BF00847316

Li, L., Li, X., \& Hu, Y. (2016). Free vibration analysis of nonlocal strain gradient beams made of functionally graded material. International Journal of Engineering Science, 102, 77-92. https://doi.org/10.1016/j.ijengsci.2016.02.010

Nazarenko, L., Khoroshun, L., Müller, W. H., \& Wille, R. (2009). Effective thermoelastic properties of discrete-fiber reinforced materials with transversally-isotropic components. Continuum Mechechanics and Thermodynamics, 20, 429-458. https://doi.org/10.1007/s00161-009-0092-6

Nazarenko, L., \& Stolarski, H. (2016a). Energy-based definition of equivalent inhomogeneity for various interphase models and analysis of effective properties of particulate composites. Composites Part B, 94, 82-94. https://doi.org/10.1016/j.compositesb.2016.03.015

Nazarenko L., \& Stolarski H. (2016b). On equivalent inhomogeneities for particles with multiple-component interphases. In K. Naumenko, \& M. Aßmus (Eds.), Advanced methods of continuum mechanics for materials and structures. Advanced Structured Materials (vol. 60, pp. 49-60). Springer, Singapore. https://doi.org/10.1007/978-981-10-0959-4_3

Nazarenko, L., Stolarski, H., \& Altenbach, H. (2016). Effective properties of short-fiber composites with Gurtin-Murdoch model of interphase. International Journal of Solids and Structures, 97-98, 75-88. https://doi.org/10.1016/j.ijsolstr.2016.07.041

Nazarenko, L., Stolarski, H., \& Altenbach, H. (2017). A model of cylindrical inhomogeneity with spring layer interphase and its application to analysis of short-fiber composites. Composite Structures, 160, 635-652. https://doi.org/10.1016/j.compstruct.2016.10.024

Nazarenko, L., Stolarski, H., \& Altenbach, H. (2018b). On modeling and analysis of effective properties of carbon nanotubes reinforced materials. Composite Structures, 189, 718-727. https://doi.org/10.1016/j.compstruct.2018.01.027

Nazarenko, L., Stolarski, H., \& Altenbach, H. (2018a). Effective thermo-elastic properties of random composites with orthotropic components and aligned ellipsoidal inhomogeneities. International Journal of Solids and Structures, 136-137, $220-240$. https://doi.org/10.1016/j.ijsolstr.2017.12.016

Seidel, G. D., \& Lagoudas, D. C. (2006). Micromechanical analysis of the effective elastic properties of carbon nanotube reinforced composites. Mechanics of Materials, 38(8-10), 884-907. https://doi.org/10.1016/j.mechmat.2005.06.029

Song, Y. S., \& Youn, J. R. (2006). Modelling of effective elastic properties for polymer based carbon nanotube composites. Polymer, 47(5), 1741-1748. https://doi.org/10.1016/j.polymer.2006.01.013

Tserpes, K. I., \& Silvester, N. (2014). Modeling of carbon nanotubes, graphene and their composites. Springer Series in Materials Science (vol. 188). https://doi.org/10.1007/978-3-319-01201-8_10

Xu, F., Zhang, X., \& Zhang, H. (2018). A review on functionally graded structures and materials for energy absorption. Engineering Structures, 171, 309-325. https://doi.org/10.1016/j.engstruct.2018.05.094 